\title{
SPH Radiative Hydrodynamics Methods
}

\author{
Hajime Susa \\ Departiment of Physics, Konan University, Kobe, Japan \\ email: susa@konan-u.ac.jp
}

\begin{abstract}
In this paper, we review the radiative hydrodynamics methods based upon Smoothed Particle Hydrodynamics(SPH). There are already various implementations so far, which can be categorized into three types: moment equation solvers, Monte Carlo methods, and ray-tracing schemes. These codes have been applied to various astrophysical problems including dynamics of dense proto-stellar cores, photoionization feedback of massive stars on molecular clouds, radiative feedback in the early universe, etc. Among these different methods, we focus on the ray-tracing schemes. We also describe one particular ray-tracing code RSPH in some details.
\end{abstract}

Keywords. methods: numerical, radiative transfer, hydrodynamics

\section{Various astrophysical problems and different codes}

Smoothed Particle Hydrodynamics(SPH) is the most widely used Lagrangian scheme for the studies of star formation and galaxy formation. Thanks to its Lagrange nature, it has an advantage over the Eulerian grid codes in resolving the collapsing regions which are always found in numerical simulations of star/galaxy formation. On the other hand, radiative processes including radiative cooling/heating, radiation pressure, photo-chemical reactions, play central roles in star/galaxy formation. Thus, implementing radiation physics in $\mathrm{SPH}$ is quite important and a natural pathway to tackle such astrophysical problems.

A number of authors have already developed radiative hydrodynamics codes with SPH. Most of the codes use approximations for radiation transfer calculations depending on the problem which they try to solve. Studies of the formation of proto-stellar cores have the longest history of this type of numerical simulations. Because of the relatively large optical depth in the dense collapsing core, the diffusion/flux limited diffusion approximation has been used in these studies (Lucy 1977, Brookshaw 1994, Whitehouse et al. 2005, Viau et al. 2006, Mayer et al. 2007, Price \& Bate 2009). Flux limited diffusion is also used in the studies of supernova explosions(Herant \& Woosley 1994, Fryer et al. 2006), where the system is also very dense, so optically thick. The advantage of the diffusion/flux limited diffusion approximation is that it is much less computationally costly than direct methods like Monte Carlo schemes. It utilizes the moment equations of the radiation transfer equation, with a simple closure relation between the mean intensity and pressure tensor of the radiation field. Thus, the five dimensional radiation transfer equation is reduced to equations for three dimensional variables like energy density or radiation flux. As a result, the cost of the computation is reduced dramatically. Another type of moment equation solver is called Optically Thin Variable Eddington Tensor (OTVLT) scheme developed by Gnedin \& Abel (2001). In this scheme, the variable Eddington tensor is calculated under the assumption that the system is optically thin, whereas the diffusion approximation is basically applicable in the optically thick region. OTVLT has been coupled with SPH (Gadget) by Petkova \& Springel (2009), and was applied to cosmic reionization problem.

Apart from these studies with moment equation solvers, there is another direction for the development of radiative hydrodynamics with SPH. In case we want to obtain 
realistic images or the spectra of proto-stellar cores from theoretical calculations, rough approximations with moment equation solvers are not good enough to be compared with observations. In these cases, Monte Carlo schemes are often used, which have also been implemented in SPH density fields (Oxley \& Woolfson 2003, Stamatellos \& Whitworth 2005, Forgan \& Rice 2010). If we could use enough photon packets in a simulation, the outcome of the calculation approaches the precise solution, while the computational cost becomes very large. So, most of the Monte Carlo solvers at present are used for post-processing the results of hydrodynamical calculations. Monte Carlo schemes with SPH are also widely used in studies of cosmic reionization (Semelin et al. 2007, Pawlik \& Schaye 2008, Altay et al. 2008, Maselli et al. 2009), in which large scale ionization pattern is not significantly changed by the coupling of radiation transfer and hydrodynamics.

On the other hand, if we are interested in much smaller scales $\lesssim 10 \mathrm{kpc}(\simeq 10 \mathrm{~km} / \mathrm{s} \times$ $10 \mathrm{Gyr})$, radiative hydrodynamical effects come into play. For instance, if we consider the radiative feedback effects on the formation of the first galaxies, or the first/second generation of stars, intense radiation flux from neighboring sources can ionize and heat up the gas (e.g. Susa \& Umemura 2004, 2006). Another important example is the positive/negative radiative feedback by massive stars in local star formation processes (e.g. Bisbas et al. 2009, Gritschneder et al. 2009). In order to tackle these problems, a careful treatment of photoionization as well as a radiation transfer solver fully coupled with hydrodynamics is required. Because of the too heavy cost of Monte Carlo radiation transfer coupled with hydrodynamics, several authors have developed a different type of radiation transfer solver, those are categorized as ray-tracing codes. In these codes, the transfer of diffuse photons is not solved. These photons are assumed to be absorbed "on-the-spot" and never scattered or re-emitted to a spatially distant position. Thus, we only have to solve the transfer of photons directly from the source star under such approximation. As a result, the computational cost of the radiation transfer calculation is greatly reduced. In this paper, we focus on the description of such ray-tracing schemes. We also describe in some detail one particular ray-tracing scheme RSPH developed by ourselves.

\section{Ray-Tracing schemes coupled with SPH}

\subsection{Ray-Tracing}

The core part of the ray-tracing scheme is the optical depth integrator. As for the method to calculate the optical depth, the codes developed so far are basically divided into two categories. the first contains the "neighbor connecting" schemes (Kessel-Deynet \& Burkert 2000, Susa \& Umemura 2004, Susa 2006, Miao et al. 2006, Dale et al. 2007, Yoshida et al. 2007, Gritschneder et al. 2009). In these schemes the optical depth from the point source is assessed utilizing the neighbor lists of SPH particles. Although there are some variations in the usage of the neighbor lists, all codes basically follow the neighbor lists along the light rays from the sources to all SPH particles to create the evaluation points of the gas density and optical depth on the rays. Summing up all the contributions of SPH particles on the evaluation points of the light rays, we can evaluate the optical depth from the source stars to each SPH particle.

On the other hand, a few codes (Alvarez et al. 2006, Bisbas et al. 2009) utilize a public domain code called HEALPix (Górski et al. 2005). HEALPix is a software which produces a subdivision of a spherical surface in which each pixel covers the same surface area as every other pixel. In these codes, light rays are generated corresponding to the small solid angles provided by HEALPix. Then, the SPH density field is mapped to the grids along these rays to calculate the optical depth. Another advantage of HEALPix is its 
hierarchical structure. The light ray from the source can be split as the distance from the source becomes large, based on the tree structure of solid angles in HEALPix(Abel \& Wandelt 2002). Such a ray-splitting procedure reduces the computational cost dramatically.

\subsection{Photoionization solver}

Since most of the ray-tracing schemes are designed to investigate the radiative feedback from the photoionization process, it is crucial to implement a photoionization solver in the code.

The photoionization rate of $\mathrm{HI}$ and the photoheating rate for each SPH particle labeled as $i$ are given by

$$
\begin{aligned}
k_{\mathrm{ion}}^{(1)}(i) & =n_{\mathrm{HI}}(i) \int_{\nu_{L}}^{\infty} \int \frac{I_{\nu}(i)}{h \nu} \sigma_{\nu} d \Omega d \nu, \\
\Gamma_{\mathrm{ion}}^{(1)}(i) & =n_{\mathrm{HI}}(i) \int_{\nu_{L}}^{\infty} \int \frac{I_{\nu}(i)}{h \nu} \sigma_{\nu}\left(h \nu-h \nu_{L}\right) d \Omega d \nu .
\end{aligned}
$$

Here $n_{\mathrm{HI}}(i)$ represents the number density of neutral hydrogen of the $i$-th particle, and $\sigma_{\nu}$ is the photoionization cross section. The frequency at the Lyman limit is denoted by $\nu_{\mathrm{L}}$, and $\Omega$ is the solid angle. $I_{\nu}(i)$ is the intensity of the ultraviolet radiation that irradiates the $i$-th particle, which is obtained by the ray-tracing discussed in the previous subsection.

In case the optical depth for a single SPH particle is less than $\sim O(1)$, equations (2.1) and (2.2) are valid. If the optical depth becomes much larger than unity, however, those expressions could lead to essentially zero ionization and heating rates because the equations do not conserve the number of photons numerically.

There are two directions to avoid this difficulty. The first one is the "photon conserving method" (Kessel-Deynet \& Burkert 2000, Susa 2006, Miao et al. 2006, Gritschneder et al. 2009) similar to the scheme developed in grid codes (e.g. Abel, Norman \& Madau 1999).

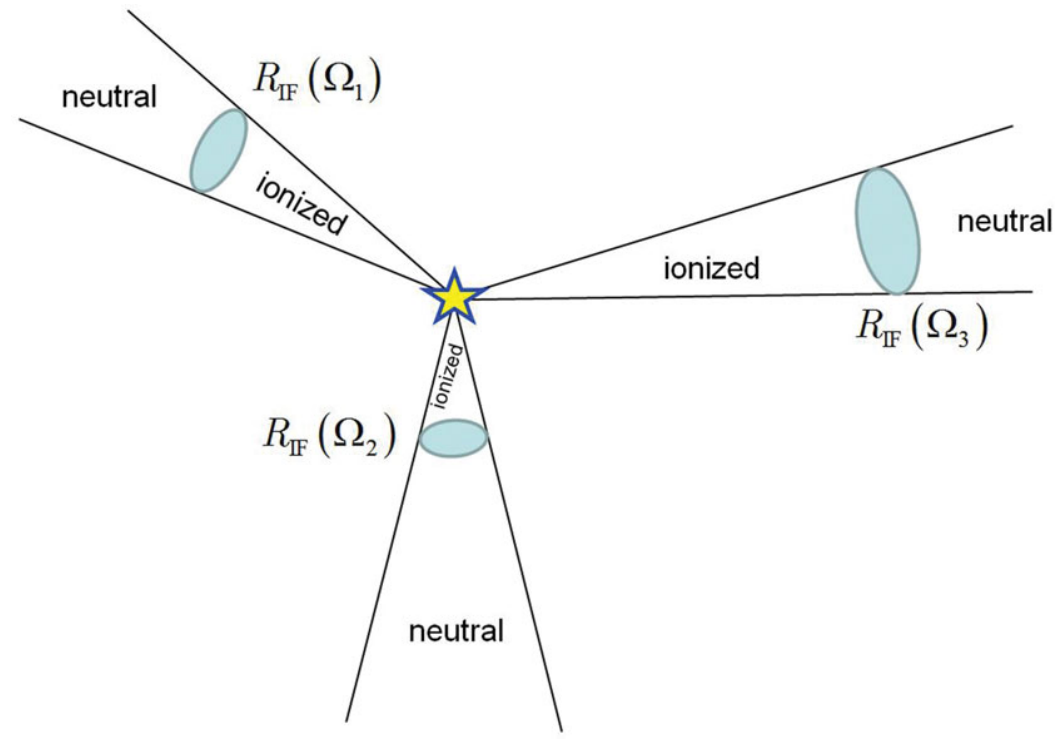

Figure 1. Schematic description of I-front tracking schemes. Here the positions of the ionization front along particular three directions corresponding to $\Omega_{1}, \Omega_{2}, \Omega_{3}$ are shown. 
We combine equations (2.1),(2.2) without the suffix $i$ and radiation transfer equation to rewrite the ionization rate and the photoheating rate as follows:

$$
\begin{aligned}
k_{\text {ion }} & =-\frac{1}{4 \pi r^{2}} \frac{d}{d r} \int_{\nu_{L}}^{\infty} \frac{L_{\nu}^{*} \exp \left(-\tau_{\nu}\right)}{h \nu} d \nu, \\
\Gamma_{\text {ion }} & =-\frac{1}{4 \pi r^{2}} \frac{d}{d r} \int_{\nu_{L}}^{\infty} \frac{L_{\nu}^{*} \exp \left(-\tau_{\nu}\right)}{h \nu}\left(h \nu-h \nu_{L}\right) d \nu,
\end{aligned}
$$

where $L_{\nu}^{*}$ denotes the intrinsic luminosity of the source and $r$ is the distance from the source. Then we integrate these rates in a small volume within $\left(r_{i}-\Delta r_{i} / 2, r_{i}+\Delta r_{i} / 2\right)$ to obtain the "volume averaged" rates:

$$
\begin{aligned}
k_{\text {ion }}^{(2)}(i) & \equiv \overline{k_{\text {ion }}}=\frac{3}{\Delta r_{i}} \frac{\Phi_{1}\left(r_{i}-\Delta r_{i} / 2\right)-\Phi_{1}\left(r_{i}+\Delta r_{i} / 2\right)}{3 r_{i}^{2}+\Delta r_{i}^{2} / 4}, \\
\Gamma_{\text {ion }}^{(2)}(i) & \equiv \overline{\Gamma_{\text {ion }}}=\frac{3}{\Delta r_{i}} \frac{\Phi_{2}\left(r_{i}-\Delta r_{i} / 2\right)-\Phi_{2}\left(r_{i}+\Delta r_{i} / 2\right)}{3 r_{i}^{2}+\Delta r_{i}^{2} / 4}
\end{aligned}
$$

where

$$
\begin{aligned}
\Phi_{1}(r) & =\int_{\nu_{L}}^{\infty} \frac{L_{\nu}^{*}}{4 \pi} \frac{\exp \left(-\tau_{\nu}\right)}{h \nu} d \nu \\
\Phi_{2}(r) & =\int_{\nu_{L}}^{\infty} \frac{L_{\nu}^{*}}{4 \pi} \frac{\exp \left(-\tau_{\nu}\right)}{h \nu}\left(h \nu-h \nu_{L}\right) d \nu .
\end{aligned}
$$

Here $r_{i}$ is the distance between the source and $i$-th particle, $\Delta r_{i}$ denotes the spatial step of the ray-tracing integration. Using these volume averaged photoionization rates, we can solve the time dependent rate equations of chemical species by ordinary implicit time integration. The method described above has the important advantage that the propagation of the ionization front is properly traced even for a large particle separation with optical depth greater than unity.

Another approach to overcome the difficulty of the ionizing photon transfer is called "Ifront tracking method" or "Strömgren volume approximation" (Alvarez et al. 2006, Dale et al. 2007, Yoshida et al. 2007, Bisbas et al. 2009). This method basically tries to follow the position of ionization front (I-front) for every direction (Fig.1). The equation used to follow the evolution of I-front is

$$
\frac{d R_{\mathrm{IF}}}{d t}=\frac{Q\left(R_{\mathrm{IF}}, t\right)}{4 \pi R_{\mathrm{IF}}^{2} n_{\mathrm{HI}}\left(R_{\mathrm{IF}}, t\right)}
$$

where

$$
Q(R, t)=Q_{*}-4 \pi \alpha_{B} \int_{0}^{R} r^{2} n^{2}(r, t) d r
$$

Here $Q_{*}$ is the number of ionizing photons emitted by the source, while $Q$ denotes the number of photons per unit time not consumed by the case B recombination process within the ionized region. Thus, $Q / 4 \pi R^{2}$ is the flux of ionizing photons at the I-front, which should balance the flux of neutral hydrogen streaming into the HII region over the I-front. Consequently, we obtain the evolutionary equation of the I-front position $R_{\mathrm{IF}}$. The computational cost of this approximation is smaller than the previous method discussed in the first half of this subsection, because this scheme does not solve the local photoionization rate equations. However, this also could be a disadvantage, since it is not possible to trace the photoheating beyond the I-front, which could be important in case the spectrum of the radiation source is very hard like for first stars or QSOs (e.g. Susa \& Umemura 2006). 


\section{RSPH}

\subsection{Brief description of the code}

In this section, we briefly describe the ray-tracing code RSPH which is developed by ourselves. The code was originally designed to investigate the formation and evolution of the first generation of objects at $z \gtrsim 10$, where the radiative feedback from various sources plays important roles. The code can compute the fraction of chemical species e, $\mathrm{H}^{+}, \mathrm{H}, \mathrm{H}^{-}, \mathrm{H}_{2}$, and $\mathrm{H}_{2}^{+}$by fully implicit time integration. It also can deal with multiple sources of ionizing radiation as well as radiation at the Lyman-Werner band. We use the version of SPH by Umemura(1993) with modifications according to Steinmetz \& Müller(1993), and also adopt the particle resizing formalism by Thacker et al. (2000).

The non-equilibrium chemistry and radiative cooling for primordial gas are calculated by the code developed by Susa \& Kitayama(2000), where the $\mathrm{H}_{2}$ cooling and reaction rates are mostly taken from Galli \& Palla (1998).

As for the photoionization process, we employ the on-the-spot approximation (Spitzer 1978) which has already been discussed in section 1 . We solve the transfer of ionizing photons directly from the source, whereas we do not solve the transfer of diffuse photons. Instead, it is assumed that the recombination photons are absorbed in the neighborhood of the spatial position where they are emitted. Because of the absence of the source term in this approximation, the radiation transfer equation becomes very simple. The method to solve the transfer equation reduces to the simple problem of assessing the optical depth from the source to every SPH particle.

The optical depth is integrated utilizing the neighbor lists of SPH particles. In our scheme, we do not create many grid points on the light ray. Instead, we just create one grid point per SPH particle in its neighborhood. In Fig.2, the scheme is schematically shown for a particular case. In case we try to assess the optical depth at the particle labeled as P0, we find the 'upstream' particle for P0 on its line of sight to the source. In this case, the 'upstream' particle is P2. The selection criterion of the 'upstream' particle is that it has the smallest angle $\theta$ (see Fig.2) among the particles in the neighbor list of P0. Then the optical depth from the source to P0 is obtained by summing up the optical depth at P2 and the differential optical depth between P0 and P2. Remark that the the differential optical depth $d \tau_{\mathrm{P} 0}$ is measured from $\mathrm{P} 0$ to the point on the light ray

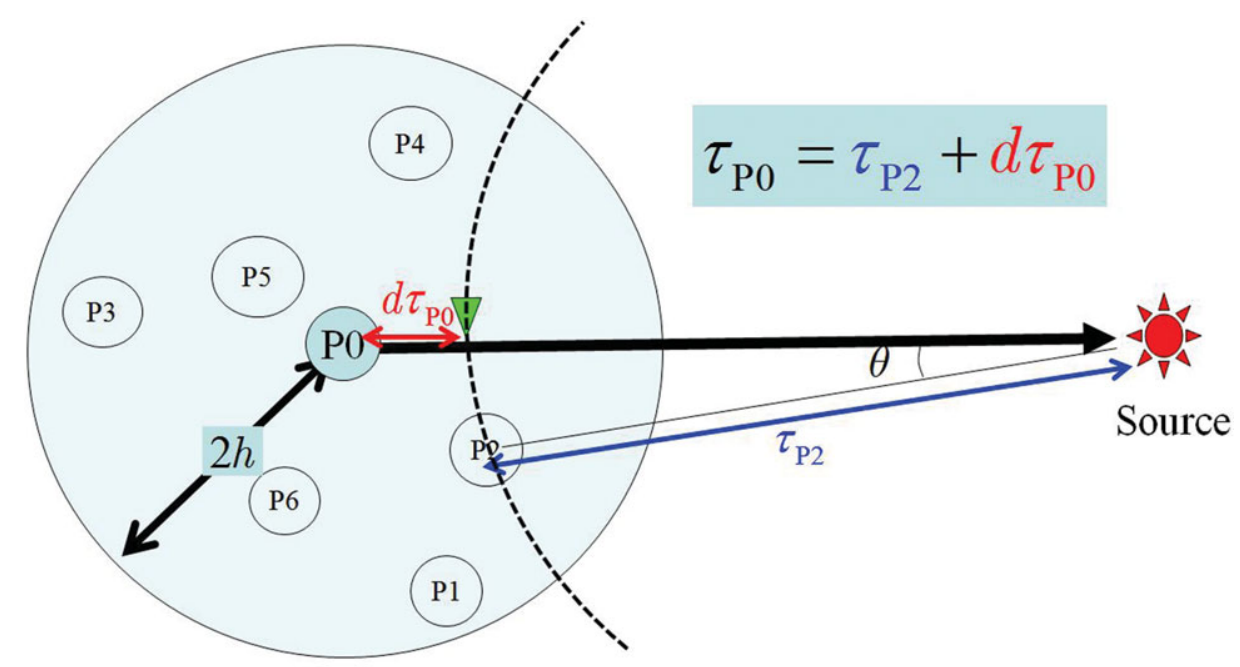

Figure 2. Schematic description of the ray-tracing procedure of RSPH. 
marked by the wedge in Fig.2. The distance between the wedge and the source is set to equals the interval between P2 and the source. Thus, the geometrical prolongation of path length due to the deviation from the straight line is avoided. It is also worth noting that this procedure naturally introduces ray-splitting. The number of light rays in the neighborhood of the source should be similar to the number of neighbor particles of the source, while each SPH particle has its own ray to the source. Thus, the rays from the source are split to reach many SPH particles.

The code is already parallelized with the MPI library. The computational domain is divided by the so called Orthogonal Recursive Bisection method. The parallelization method for the radiation transfer part is similar to the Multiple Wave Front method developed by Nakamoto, Umemura, \& Susa (2001) and Heinemann et al. (2006), but it is changed to fit to the SPH code. The details are described in Susa (2006). The code is also able to handle self-gravity with a Barns-Hut tree, which is also parallelized. We also remark that the code has already been applied to various astrophysical problems including radiative feedback during star formation / galaxy formation in the early universe (e.g. Susa \& Umemura 2006, Susa 2008).

\subsection{Test simulations}

We have performed various tests with the code. Among several test calculations, here we show the results of most simple and fundamental tests. The test results are compared with the numerical solutions from one dimensional radiation hydrodynamic simulations. In this test calculation, we use 8 nodes ( 16 Xeon processors with gigabit ethernet) and $1048576 \mathrm{SPH}$ particles. The clock time of this particular run is about 3 hours.

We put a single source at the center of an uniform gas cloud and trace the propagation of the ionization front. The initial number density of the gas is $n_{\mathrm{H}}=0.01 \mathrm{~cm}^{-3}$, and the temperature is $T=3 \times 10^{2} \mathrm{~K}$. The ionizing photon luminosity of the source is $S=$ $1.33 \times 10^{50} \mathrm{~s}^{-1}$ and the spectrum is black body with $T_{*}=9.92 \times 10^{4} \mathrm{~K}$, which is typical for POPIII stars. This parameter set is chosen so that the we can trace the well known transition of the ionization front from R-type to D-type in uniform media.

Figure 3 shows the spatial distributions of density and temperature at different times ( $\left.t=10^{7} \mathrm{yr}, 10^{8} \mathrm{yr}, 10^{9} \mathrm{yr}\right)$. The results are compared with highly accurate one dimensional simulation. At $10^{7} \mathrm{yr}$, no hydrodynamical change of gas density is found (left panel), since the ionization front is still R-type. The temperature distribution shows that the gas close to the source is already heated to $\sim 10^{4} \mathrm{~K}$ (right panel). Between $10^{7} \mathrm{yr}$ and $10^{8} \mathrm{yr}$, the type of the ionization front changes from $\mathrm{R}$ to $\mathrm{D}$, followed by the formation of less dense cavity around the central source. At $10^{9} \mathrm{yr}$, a clear dense shell is formed at the ionization front, which is the typical aspect of the D-type front. Slight scatter and deviation from the 1D simulation are found in the spatial distribution, especially at later epoch. However, the agreement between the two results is acceptable.

We also point out that RSPH has taken part in the Cosmological Radiation Transfer Comparison Project (Iliev et al. 2006, Iliev et al. 2009), where it was compared with many other codes including several grid codes. Although we found good agreements with the results from various other codes, there are slight disagreements for a particular problem. $\mathrm{RSPH}$ is basically very close to the $1 \mathrm{D}$ results for spherical problems, however, it slightly deviates from other results for plane parallel calculations with the shadow casted by dense cloud (Tests 3 and 7). There are mainly two reasons: 1) RSPH uses 'glass' initial particle distribution in order to realize the uniform density of the media, whereas the grid codes use the grid parallel to the light ray. 2) RSPH cannot avoid the 'diffusion of optical depth' because of its ray-tracing scheme. Thus, it is difficult to obtain very sharp 

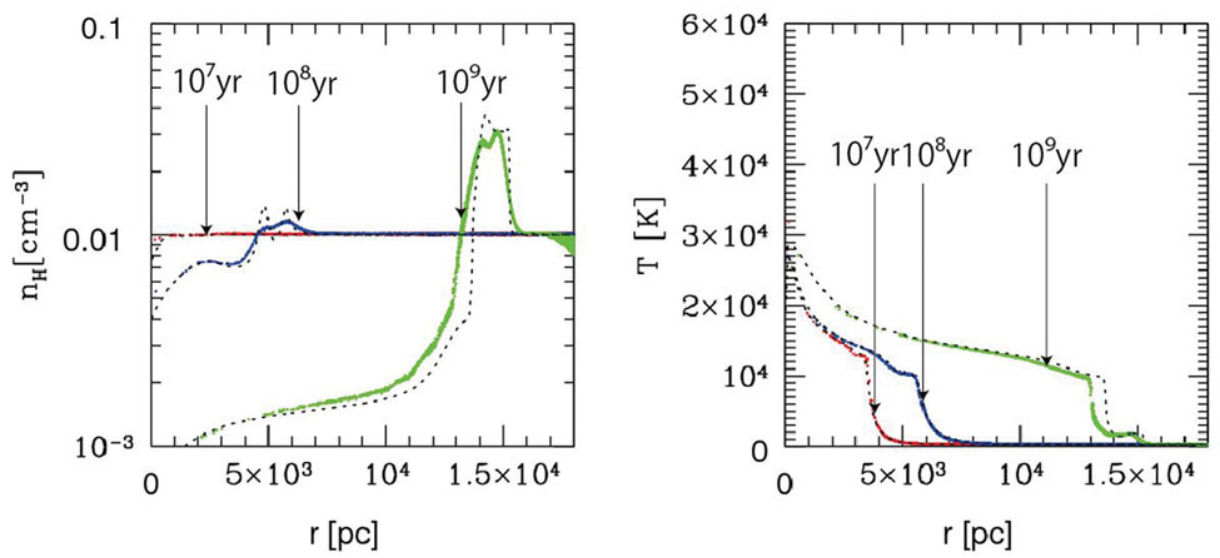

Figure 3. Spatial distribution of physical quantities at three times $\left(t=10^{7}, 10^{8}, 10^{9} \mathrm{yr}\right)$ are shown for the test run. The two panels show the density and temperature as functions of the distance from the source. The dotted lines denote the results from the 1D simulation.

boundaries between the shadow and the ionized region. We have to keep in mind this disadvantage of RSPH scheme.

\section{Summary}

In this paper, we reviewed the radiative hydrodynamics methods coupled with SPH. Previous implementations can be categorized into three types: moment equation solvers, Monte Carlo methods, and ray-tracing schemes.

The core part of the ray-tracing schemes consists of an optical depth integrator and a photoionization solver. As for the optical depth integration, most of the codes employ "neighbor connecting" schemes, whereas a few utilize HEALPix to generate hierarchical light rays. There are two basic types of photoionization solvers: the simple photon conserving scheme and I-front tracking method. Both of them are useful to trace the photoionization, however, in case we have to consider photoheating beyond the I-front, the I-front tracking method is not a good approximation.

We also described the ray-tracing code RSPH in some detail. The results of the test calculations agree well with the results from $1 \mathrm{D}$ simulations and various other radiative hydrodynamics codes including grid codes.

\section{Acknowledgements}

HS thanks M. Umemura for insightful comments on the construction of RSPH code. HS also thanks all the collaborators in the Cosmological Radiation Transfer Code Comparison Project for fruitful discussions during the three workshops in CITA, Leiden and Austin. The analysis has been made with computational facilities at the Center for Computational Science at the University of Tsukuba and Rikkyo University and Konan University. This work was supported in part by the Ministry of Education, Culture, Sports, Science, and Technology (MEXT), Grants-in-Aid, Specially Promoted Research 16002003, Scientific Research (C), 22540295.

\section{References}

Abel T., Norman M. L., \& Madau P., 1999, ApJ, 523, $66+$

Abel, T. \& Wandelt, B. D. 2002, MNRAS, 330, L53 
Altay, G., Croft, R. A. C., \& Pelupessy, I. 2008, MNRAS, 386, 1931

Alvarez, M. A., Bromm, V., \& Shapiro, P. R. 2006, ApJ, 639, 621

Bisbas, T. G., Wünsch, R., Whitworth, A. P., \& Hubber, D. A. 2009, A\&\&A, 497, 649

Brookshaw, L. 1994, Memorie della Societa Astronomica Italiana, 65, 1033

Dale, J. E., Ercolano, B., \& Clarke, C. J. 2007, MNRAS, 382, 1759

Forgan, D. \& Rice, K. 2010, MNRAS, 1049

Fryer, C. L., Rockefeller, G., \& Warren, M. S. 2006, ApJ, 643, 292

Galli, D. \& Palla, F. 1998, A\&3A, 335, 403

Gnedin, N. Y. \& Abel, T. 2001, New Astronomy, 6, 437

Górski, K. M., Hivon, E., Banday, A. J., Wandelt, B. D., Hansen, F. K., Reinecke, M., \& Bartelmann, M. 2005, ApJ, 622, 759

Gritschneder, M., Naab, T., Burkert, A., Walch, S., Heitsch, F., \& Wetzstein, M. 2009, MNRAS, 393, 21

Herant, M. \& Woosley, S. E. 1994, ApJ, 425, 814

Heinemann, T., Dobler, W., Nordlund, Å., \& Brandenburg, A. 2006, A\&A, 448, 731

Iliev, I. T., et al. 2006, MNRAS, 371, 1057

Iliev, I. T., et al. 2009, MNRAS, 400, 1283

Kessel-Deynet, O. \& Burkert, A. 2000, MNRAS, 315, 713

Lucy, L. B. 1977, AJ, 82, 1013

Maselli, A., Ciardi, B., \& Kanekar, A. 2009, MNRAS, 393, 171

Mayer, L., Lufkin, G., Quinn, T., \& Wadsley, J. 2007, ApJL, 661, L77

Miao, J., White, G. J., Nelson, R., Thompson, M., \& Morgan, L. 2006, MNRAS, 369, 143

Nakamoto, T., Umemura, M., \& Susa, H. 2001, MNRAS, 321, 593

Pawlik, A. H. \& Schaye, J. 2008, MNRAS, 389, 651

Petkova, M. \& Springel, V. 2009, MNRAS, 396, 1383

Price, D. J. \& Bate, M. R. 2009, MNRAS, 398, 33

Oxley, S. \& Woolfson, M. M. 2003, MNRAS, 343, 900

Semelin, B., Combes, F., \& Baek, S. 2007, A\& A, 474, 365

Spitzer, L. Jr. 1978, in Physical Processes in the Interstellar Medium (John Wiley \& Sons, Inc. 1978)

Stamatellos, D., \& Whitworth, A. P. 2005, A\& A, 439, 153

Steinmetz, M. \& Müller, E. 1993, A\&A, 268, 391

Susa, H. 2006, PASJ, 58, 445

Susa, H. \& Kitayama, T. 2000, MNRAS, 317, 175

Susa, H. \& Umemura, M. 2004, ApJ, 600, 1

Susa, H. \& Umemura, M. 2006, ApJL, 645, 93

Susa, H. 2008, ApJ, 684, 226

Thacker, J., Tittley, R., Pearce, R., Couchman, P. \& Thomas, A. 2000, MNRAS 319, 619

Umemura, M. 1993, ApJ, 406, 36

Viau, S., Bastien, P., \& Cha, S.-H. 2006, ApJ, 639, 559

Whitehouse, S. C., Bate, M. R., \& Monaghan, J. J. 2005, MNRAS, 364, 1367

Yoshida, N., Oh, S. P., Kitayama, T., \& Hernquist, L. 2007, ApJ, 663, 68 